\title{
Asdic to sonar
}

R.V. Jones

Seek and Strike: Sonar, Anti-Submarine Warfare and the Royal Navy 1914-54. By Willem Hackmann.

Her Majesty's Stationery Office: 1984. Pp.487. £15.95.

THE detection and location of submarines in the sea has one factor in common with the analogous processes associated with finding aircraft in the air - the transmission of wave-borne energy. But while the air is a nearly uniform and transparent medium to electromagnetic radiation, and while an aircraft is a palpable discontinuity in that medium, the sea is effectively opaque over almost the entire range of the radio spectrum. It was therefore quickly realized that the detection of submarines would have to depend on acoustic waves - mechanical waves which need transducers for their generation and reception, a further contrast with radar, where radio waves are launched from and received by antennae which are simple extensions of electronic circuitry. As a result, once the problem of aircraft detection was formulated, the radar solution was brilliantly found and exploited to such an extent that in the decade 1935-1945 almost all the essential techniques had been developed.

No such exhilarating story can be told of submarine detection. Instead, it has provided one of the greatest challenges to the application of physics, where ground has been won only by prolonged effort. Seek and Strike records what was done in Britain between 1914, when the impact of the U-Boats was first felt, and 1954, when submarine warfare entered a new phase with the launching of the first nuclear submarine - the USS Nautilus.

The author, Willem Hackmann, is Assistant Curator of the Museum of the History of Science at Oxford, and he appropriately starts his account with the speculations of Aristotle and Pliny the Younger on the detection of underwater sound by fish. This has its analogy in the use of hydrophones to detect submarines by sound generated by their engines or hydrodynamically by their passage through water; this technique has been developed to detect submarines at ranges of hundreds of miles, but it is limited operationally both by the vagaries of transmission in a medium whose refractive index varies with salinity and temperature, and by a background of other noises generated not only by surface waves and ships, but also by such modest creatures as snapper shrimps.

While hydrophones can give general, and indeed impressive, warnings, ultrasonic echo detection - which gives range as well as bearing - is necessary to locate a submarine precisely enough for it to be attacked. Hackmann gives the credit for the original development of the method to Paul Langevin, but it may be worth mentioning that James Wilson in his recent biography suggests that some of it should also go to Rutherford.

The author has clearly done a great deal of painstaking work in pursuing the many aspects of a long and involved story, and in refining them into a readable and admirably documented account. Several points emerge from it. First, despite the fact that the Royal Navy appreciated as long ago as 1873 the importance of science when it founded the Royal Naval College at Greenwich to ensure that naval officers would study both science and mathematics, and despite the enthusiasm for science and engineering shown by Admiral John Fisher, few naval officers were prepared to take scientists fully into their confidence, even when faced with the U-Boat threat. Instead of letting scientists such as Rutherford experience the conditions of hunting U-Boats at sea for themselves, the chief naval liaison officer demurred, saying that all that the scientists need be told "was that the enemy submarines were in the sea and that means were required to detect their presence". Only after the advantages of close cooperation between scientists and serving officers had been demonstrated in air defence in the Second World War were the Services completely won over.

Another feature of the development of Asdic (or Sonar) was the continuous battle between long and short term interests: should greater priority be given to the development of a weapon for tomorrow or to research that might provide a much better weapon the day after tomorrow? The right answer depends on circum- stances; but in naval weapons, such as Asdic or signalling, the answer was more often than not determined in favour of the short term, because appointments in the Navy have characteristically a two-year duration, and so captains of establishments such as The Signals School tended to favour programmes from which they might expect some concrete result within their term of office.

A further message is the danger of "overselling" a weapon, causing first an unjustified reliance on its performance in operations, and then a loss of confidence when its shortcomings become clear, as exemplified by Churchill's complaint to the First Sea Lord in 1941 about Asdic ". . . the failure of our methods, about which so much was proclaimed by the Admiralty before the war". And yet another is the merit of trying a technique or system "the other way round", in this case the firing of the depth charges ahead over the bow of the hunting ship instead of dropping them over the stern, which led to an increase in success rate from about $7 \%$ to about $30 \%$ as a proportion of kills to attacks.

All these lessons, which have relevance far beyond submarine warfare alone, are to be found in the book. Alongside them are such morsels as the attempts of 1915 to train seagulls to hunt for U-Boat periscopes; the renaming of the quartz crystals required for piezoelectric transducers as "asdevite" to avoid disclosing their composition, and the recruitment of a firm of tombstone-makers to cut the crystals; and the fact that even a noisy submarine radiates no more energy than would light a 3-volt torch bulb.

Seek and Strike tells a story of successes tempered with failures in the sustained application of physics to one of the most crucial problems of warfare this century. It was well worth writing - and is well worth reading - both for its significance as history and for the lessons it exemplifies.

R.V. Jones is Emeritus Professor of Natural Philosophy at the University of Aberdeen and Chairman of the Defence Committee of the Centre for Policy Studies, London.

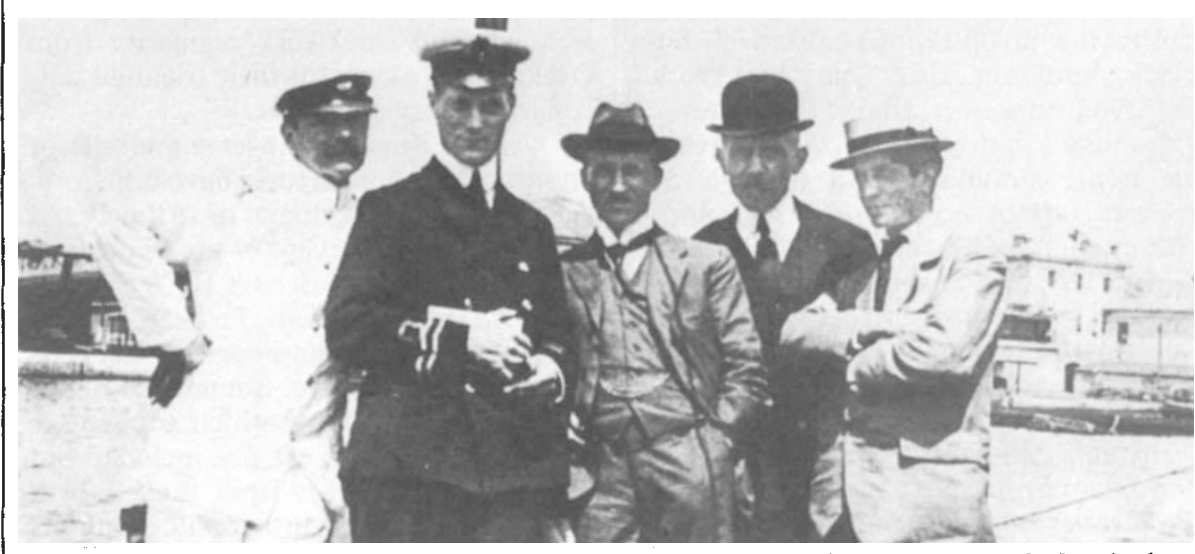

Pioneers of ultrasonic echo ranging; Paul Langevin is fourth from the left, wearing the bowler hat. The photograph was probably taken at Toulon in May 1918. 